\title{
Chapter 14 Conflict Resolution with Agents in Smart Cities
}

\author{
Pablo Chamoso \\ University of Salamanca, Spain \\ Fernando De la Prieta \\ University of Salamanca, Spain
}

\author{
Javier Bajo Pérez \\ Polytechnic University of Madrid, Spain \\ Juan Manuel Corchado Rodríguez \\ University of Salamanca, Spain
}

\begin{abstract}
Today, there is a common trend to use tools and methodologies that allow the development of MultiAgent Systems (MAS) with capabilities of reorganization and adaptation to determine changes in their environments. This work presents an architecture based on different levels and whose key level is the one corresponding to the semi-open type of MAS, structured in such a way that it is able to solve conflicts. In addition, a case study is introduced with the objective of showing the possibilities on conflict resolution basis, where a specifically designed architecture is utilized for that purpose. In particular, the system is applied to the resolution of the conflict raised by the decision of the technology to be used in order to obtain or to measure information in smart cities.
\end{abstract}

\section{INTRODUCTION}

Recent technological developments have shown a rapid evolution in wireless communications, sensors, information distributed processing, and other new technologies, all of which are mainly associated to a new paradigm known as the Internet of Things (Weber, 2010, pp. 23-30). This technological evolution has resulted in an increase in the features and services available through the Internet. Accordingly, there is not only an increase in online services, but also an increase in new services in the real world, mainly in large urban areas, which use the technological base provided by the Internet of Things paradigm. In effect, we are talking about smart cities, which have emerged as a response to the challenges experienced by cities in meeting objectives regarding the socio-economic development and quality of life of their citizens (Schaffers et al., 2011, pp. 431-446).

DOI: $10.4018 / 978-1-5225-0245-6 . c h 014$

Copyright $\odot$ 2016, IGI Global. Copying or distributing in print or electronic forms without written permission of IGI Global is prohibited. 
Smart cities have become quite significant, and are currently one of the main objectives of research in America as well as in the European Union (Caragliu, Del Bo, \& Nijkamp, 2011, pp. 65-82). The main interest currently consists of directly or indirectly increasing the well-being of the population. Direct measures include offering new services or improving existing ones. Some of the ways to achieve this objective consist of tele-care services for the elderly or dependent persons, the use of traffic optimization techniques, actively controlling energy supplies, etc. Indirect measures include offering the same services, as perceived by the citizens, but changing the way they are managed or their internal performance in order to increase the economic savings of the city. This will definitely imply an indirect increase in the quality of life for the citizens (tax savings, new services offered as a result of the economic savings, etc.). An example of the latter would be the replacement of older light bulbs in public light fixtures with new bulbs with a lower power requirement (LED bulbs for instance). Thus, new technological advances make it possible to rapidly increase the ability to enhance the social well-being of a city. While this may result in greater benefits for the cities, it is not without some disadvantages.

The reasons why emerging technologies are a major benefit are self-evident: the majority of the advances are going to be useful to improve any factor, whether in data acquisition, information transmission or information processing. However, the unstoppable technological evolution means that in most of the cases different alternative technologies, with similar characteristics and capabilities, will be required to cohabitate at the same time and in the same environment. On the other hand, the constantly evolving technology makes it necessary, in some cases, to dismiss the existing infrastructure.

Thus, one of the main current concerns for most cities is the integration of new technologies in everyday environments and their ability to improve the well-being of each citizen. With the objective of solving the problems associated with the technological evolution, we turn to Agent technologies, in particular, those following a design model based on roles, objectives and norms.

There is no doubt that Multi-Agent Systems (MAS) have become tremendously significant during the last years within the environment of Distributed Artificial Intelligence (DAI). MAS allow solving problems in a distributed manner by taking advantage of social behaviors as well as the individual behavior of the agents. Since a smart city requires the features of an open system (dynamic, heterogeneous and with uncertainty), MAS have been applied in this environment with promising results, for example to address multiple aspects in the management of smart cities (Roscia, Longo, \& Lazaroiu 2013, pp. 371-376).

Recently, the design theories of MAS promote their grouping into organizations or societies, thus sharing features, norms and objectives. These societies can be open or closed societies depending on their flexibility to admit new members. These capabilities, similar to those of human societies, are perfectly suited to the new open context of smart cities. One example of these capabilities is the use of a semiopen society, as the design model would be able to evolve and auto adapt to new situations. Another example is allowing different members, independently of the technology used, to enter the society to provide the services of a specific role. For example, given the task of regulating city traffic, two roles, which would be technologically independent, might include counting the number of vehicles on the road and regulating traffic lights according to available information.

This ability would make it possible to obtain a system capable of making the best decision regarding the technologies to be used. This system also prevents possible changes from negatively impacting the management, or the services offered. This means that a change in technology will not affect the way the system uses or presents the information. It tremendously facilitates tasks requiring a human operator without needing to adapt the software that manages the information gathered by the sensors. 
This present work provides a multi-level agent-based architecture whose main functionality resides in the layer in which an organizational MAS resolves conflicts regarding the selection of the best technology at each moment. More specifically, the work focuses on the resolution of conflicts when considering the technological solution, where all the options can be valid, but only one will be the most suitable for the global system at each moment. However, the system is completely adaptable to the resolution of more conflicts related to smart cities as well as to other environments.

The rest of the section presents the current state of the art of the main technologies, theories and tools applicable to the development of the system, followed by a presentation of the main existing technology conflicts in smart cities and their potential solutions. The decisions and techniques followed to deal with this conflicts are then presented. After that, a case study, where the solution has been applied, is explained. The chapter ends by identifying certain keys required to continue the research and the conclusion to the present work.

\section{BACKGROUND}

Autonomy, robustness, flexibility and adaptability are among the most desirable characteristics with regard to the design of software applications. They are also some of the characteristics that platform must have in order to manage smart cities. To do so, there is a need for theories, models, mechanisms, methodologies and tools that can develop a system capable of reorganizing and adapting itself to possible future changes in the environment.

For this reason, the present work proposes a combination of technologies, such as organized-models based open MAS, Cloud Computing (CC) and Wireless Sensor Networks (WSN), such as the key technological context to confront the existing needs within smart cities environments.

\section{Multi-Agent Systems}

There is extensive literature about Agent Theory. For decades, the concept of agent has been that of an autonomous entity capable of interacting with the surrounding environment. However, there are some complex problems a single entity is not able to solve, and the use of a set of at least two entities collaborating to achieve a common objective is required. This set may include a MAS, which has also been extensively studied in literature (Chopra, \& Singh, 2013, pp. 101-141).

The main objective of MAS is to construct systems capable of autonomous and flexible decisionmaking, and cooperate with other systems as a society. This chapter is going to focus on the part of MAS above agents. It is commonly understood that MAS contain agents and establish relationships between them, including a set of operations that can be performed according to their observations with regards on the the external changes in the environment along the time (Ferber, 1999).

A way to analyze MAS is to compose a number of autonomous entities that interact together, or in terms of a society, an organized society of individuals, where each entity plays at least one specific role (Zambonelli, Jennings, \& Wooldridge, 2001, pp. 303-328). One of the main challenges of MAS is to both maintain the heterogeneity and the autonomy of the system components and provide guarantees about the behavior and outcomes of individual agents and of the system as whole (Vasconcelos, Kollingbaum, \& Norman, 2009, pp. 124-152). 
But beyond its definition, a MAS can be represented in different ways or have multiple configurations. Artificial societies and Virtual Organizations (VO) are important terms associated to the MAS and form the base of the current work (Zato, et al. 2012, pp. 10389-10401).

Both terms are closely related. Artificial societies can be defined as a set of interrelated and interacting artificial entities, which are governed by certain rules and conditions (Annunziato, \& Pierucci, 2003). These rules and conditions can be classified according to different criteria: openness, flexibility, stability and reliability. Openness is the possibility of an agent joining the society. Flexibility indicates the grade of constraint of the agent in its behavior due to the norms of the society. Stability measures the predictability of the actions. Finally, reliability measures the grade by which the agents can rely on the society (Davidsson, \& Johansson, 2006). From these criteria, the following types of societies can be established:

- Open Societies: Characterized by having high flexibility, which makes them a bit unstable, and not very reliable.

- Closed Societies: As opposed to the open societies, they are stable and reliable, but not flexible; that is, they are fixed or unalterable societies.

- Semi-Open Societies: The concept of gatekeeper is introduced to require external societies to communicate in advance and demonstrate they are safe to enter the system without altering the reliability and stability of the society.

- Semi-Closed Societies: These societies do not allow the authorization of external agents, but they do allow an agent of the society to perform the tasks of an external agent.

For the development of organizational systems, the most appropriate types of society would be semiopen and semi-closed, due to their inherent constraints of either flexibility or lack thereof.

Returning to the concept of organization, it can be defined as a set of entities regulated by social order mechanisms, in a way that they facilitate the achievement of common objectives. An organization must be composed of individual agents manifesting their behavior and relating to each other in a dynamic way. It can be split in sub-organizations, which can be further divided into sub-sub organizations. The behavior of these agents needs to be related, in functional terms, to the organization. Finally, its functionality has to be defined as a role to be taken into consideration within the organization (Ferber, Gutkenecht, \& Michel, 2004, pp. 65-82). The role is the key concept which represents an abstract description of the behavior of the agents. This includes their obligations, constraints and qualities. It must also include the description of the interaction procedures among the different roles that make up the system.

The chance of a conflict occurring and requiring a solution increases when agents with different roles have to cooperate and coordinate their activities. It is precisely these situations that require mechanisms to allow an efficient argument on behalf of each agent in order to make it possible to achieve an agreement.

As indicated in (Daft, 2012), decision making and the control that governs the organization follow a collaborative strategy. When a conflict occurs, each agent has to propose its solutions and attempt to find the best common solution. Each agent will try its proposed solution and must defend it before reaching the agreement. Therefore, the organizations play an important role in the resolution of conflicts. 


\section{Cloud Computing}

In recent years, the term Cloud Computing (CC) has become increasingly prevalent, especially in environments or applications where the required processing power, storage and infrastructure to supply services. CC refers to both the applications delivered as services over the Internet and the hardware capabilities and system software in the data centers that provide those services (Ambrust et al., 2010).

The theory of agents and multiagent systems (MAS) can provide a new model for the usage of CC. However, joining both computational models (MAS and CC) is a great challenge, given the difference between the two models. However, since the CC system is considered an open system and the application of MAS in open systems is a recognized challenge in which there has already been a notable rate of success. Nowadays, there is an important relationship between the way MAS work and the way in which the CC environments work. CC environments offer a high capability and high performance technology, along with high availability and scalability (De la Prieta, Rodríguez, Bajo, Corchado, 2013, pp. 37-48) (Talia, 2012), which makes it easy to apply MAS to a new group of complex applications.

The combination of CC, MAS and sensors allow to apply the solution to smart cities provides a more than adequate infrastructure (Mitton, Papavassiliou, Puliafito, \& Trivedi, 2012). First of all, CC environments can cover the computational needs for persistence of information and the computing potential that MAS require for different applications such as data mining, management of complex services, etc. The use of these technologies applied to the work described in this chapter, and as argued below, provides multiple benefits when offering cities a system capable of managing the totality of the deployed sensors and the services it offers.

\section{Wireless Sensor Networks in Smart Cities}

It may be difficult to define exactly the term 'smart city'. However, a preliminary approach could imply the use of networked infrastructure to improve economic and political efficiency and enable social, cultural and urban development (Hollands, 2008, pp. 303-320). In line with this definition, the most important technological part of smart cities is their networked infrastructure. The design of this networked infrastructure plays an important role when saving money, automating processes and determining which sensors have to be used for meeting the city goals.

The process of building automation and control systems through sensor networks, which started with wired technology, has now entered the era of wireless technology, having produced technologies such as ZigBee, Z-Wave, EnOcean, and others. WSNs are used for gathering the information needed by intelligent environments, whether in urban construction and Smart Cities, home and building automation, industrial applications or smart hospitals and so on (Liu, Seet, \& Al-Anbuky, 2013, pp. 653-674). WSNs support current requirements related to the deployment of networks that cover communication needs, and flexibly in time, space and autonomy, without requiring a fixed structure (Navarro, Bhatnagar, \& Liang, 2011, pp. 819-824). There are several wireless technologies that enable easier deployments than their wired counterparts by avoiding the need to wire buildings and decreasing the costs and drawbacks of the setup phase. However, the growing heterogeneity of this type of wireless network protocols makes it difficult to use them. WSNs make it possible to build a wide range of applications, such as the control of energy costs, monitoring environmental data, security and access control in buildings, as well as industrial and home automation, etc. Therefore, WSNs have multiple potential applications in military, industrial, environmental, biomedical and residential fields. The latter is the focus of this chapter. The 
implementation of these sensor networks tends to require sophisticated and efficient communication protocols, low-power consumption, and low cost (Akyildiz, \& Vuran, 2010). Furthermore, sensor nodes should be small and deployed in large quantities, emphasizing the need for low-cost and eco-friendly, especially in the case of smart cities.

Due to the natural evolution of technology, as a result of a vast effort in research activities and industrial investments on this field since last decade (Buratti, Conti, Dardari \& Verdone, 2009), a new conflict has emerged and is in need of a resolution. This evolution has led to the cohabitation of different technologies capable of providing valid solutions to transmit the measured information. This circumstance implies a short-term advantage for new systems, as they will always be constructed following the best option. The problem arises when maintaining those systems or when adapting working systems that are already deployed. Having to change one technology for another, seemingly better, will not provide optimal results for every case. Thus, in order to choose the best measure for the technology selection, multiple existing options must be evaluated, along with their corresponding weaknesses and strengths for the system. To do so, next section introduces an approach based on open MAS that deals with these issues.

\section{CONFLICTS IN SMART CITIES}

\section{Issues, Controversies, Problems}

Despite the ongoing efforts in the process of standardizing technologies for information and communications technology, the reality is that to date, there are multiple techniques, tools and technological protocols, which, in some cases, are not even compatible among each other. Of course, this also implies the advantage of having the opportunity to choose the technology that best suits a specific purpose, considering performance, ease of implementation, inherent characteristics, etc. However, some disadvantages can also be observed, such as the challenge of the integration and compatibility between technologies (Lian, Hsiao, \& Sung, 2013, pp. 756-767), which occurs because systems of a certain size (or even in home environments) tend to work with multiple technologies in a simultaneous way (Hafeez et al., 2014).

For an environment such as smart cities, which involve such a wide variety of information to process, the advantage of having the latest technology gets transformed into an important problem when making a decision in favor of one technology over another, especially when the system is already developed and performing, or the city is using a sensor network that has already been deployed. Thus, even though there are different inherent characteristics in technology, which could consider one of the multiple existing options to be better than the others, it does not only depend on the technology to be used, but also on external aspects.

Despite considering all the factors affecting the decision of using one technology as opposed to the others, the situation could change over time. This fact could lead to a need to partially or totally change the deployed sensor network.

Thus, the integration in the global system must first comply with the specific norms or rules for the system to admit it. In the case of the MAS, this problem is solved because it is based on a semi-open architecture that guarantees the heterogeneity of the system. So the problem disappears due to the fact any entity who wants to enter in the systems have to follow a series of the norms in order to be admitted into the system. 
In addition to integration, other problems may occur when the technology is used as the base, causing certain technologies to be discarded or chosen. These problems are influenced by factors such as the reliability of the regulatory entity for example support, API (Application Programming Interface), etc., the cost of deployment, the cost of maintenance, the consumption of resource, compatibility with already deployed infrastructures, portability, scalability or ease of use.

\section{SOLUTIONS AND RECOMMENDATIONS}

The following sub-sections present the main techniques that should be used in order to solve the problems described in the previously. So, next it is described how these techniques have been applied to the global system, which is structured in a way that follows a multi-layer, or multilevel, architecture.

\section{Global Architecture}

To achieve a better understanding of the global architecture of the system, it is necessary to have a preliminary global view of it. Each one of the levels constituting the system will be explained in detail later on. The diagram in Figure 1 shows the main layers that constitute the proposed system.

Figure 1 shows the architecture proposed to solve the problem derived from the combination or changes in technologies deployed in a smart city environment, which overcome the shortcomings of current systems. For a better understanding, the analysis starts from the lowest layers. The sensor network is deployed in the first level to gather information from the environment. This information is required to fully guarantee the services offered to citizens and allowing them to effectively become beneficiaries of the society. On top of this sensor network we can find a middleware (MW), which allows the encapsulation of this information by separating it from the used technology. Its communication protocol is also transformed into a common protocol for the system, allowing the highest levels to be able to obtain the data, no matter how they do it. Such MW and successive processing layers are executed in a CC platform that allows the system to be distributed and adaptable according to the needs of the cities. The next level, which is also executed in the CC platform of the MW, is associated to the MAS. The MAS is the most important part within the system and its objective is, depending on the situation, to act autonomously or to offer certain services the end user (software or authorized human) can use. These services constitute the last layer in the developed architecture. Next sections present in detail each of these layers and the proposed algorithm in order to resolve technological conflicts on smart cities.

\section{Sensor Network}

Starting from the lowest layer, the first level corresponds to the sensor network. This sensor network may be composed of multiple sets, possibly heterogeneous, of individual sensors. These sensors are in charge of automatically gathering the information of the nearest environment by themselves. They must also incorporate some kind of mechanism to transmit this information in order to integrate it in the global system for future use. On many occasions, the cities already use sensor networks that have 
Figure 1. Design of the multi-layer architecture that provides support for smart city management and the offered services
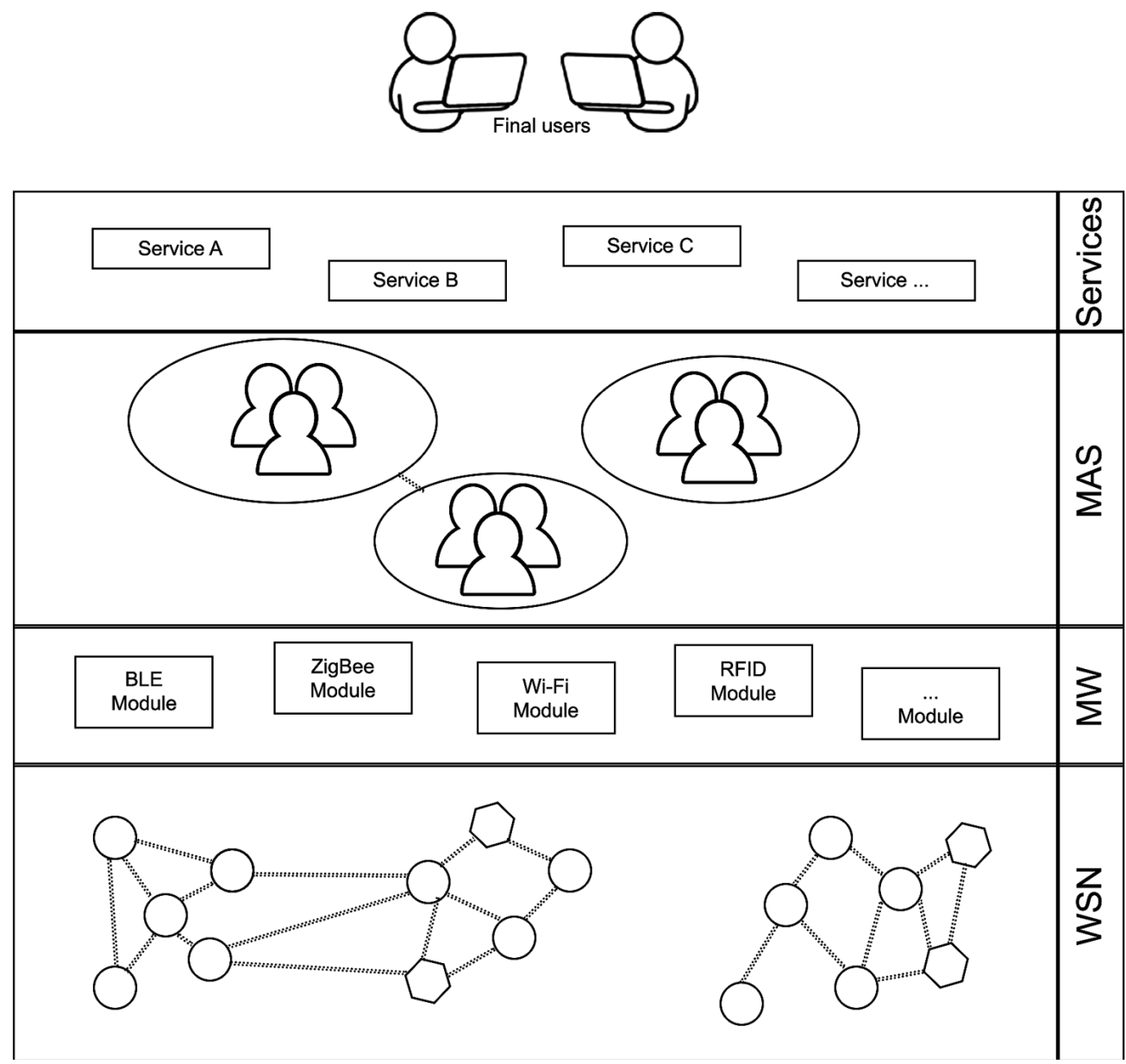

been incorporated in order to develop studies or to take measures. They could also have been added due to some other reasons, prior to the process of transforming the city into a smart city. In addition to the previously commented evolution in technology, this could result in the cohabitation of different kinds of sensors, gathering the information associated to a same environment.

To enhance its management, these sensor networks in the cities get organizationally divided into sets, where each one of them is specifically applied to a particular environment of the city. Thus, it may be the case that different sets, dedicated to a same environment, cohabitate together, which makes the system even more heterogeneous. In a practical case study, a city could require a set of sensors involved in the management of the traffic lights in the city and another set of sensors to perform vehicle-counting tasks. In such a case, both sets of sensors are involved in traffic management.

The heterogeneity of the sensor sets needs to be emphasized, as it plays an important role in this system. Similarly, as will be seen later, this structure will be associated to the roles within the MAS organizations. 


\section{Middleware}

Large-scale systems typically include MW to collect information coming from the sensors. The main task of this MW is to allow the information gathered by the sensors to be standardized within the system. To do so, the system includes an intermediate layer specialized in collecting information by using the required protocol for each technology. Once the information is obtained by the sensor and transmitted, the MW collects it and processes it (without interruption) to produce a new encapsulation to a common protocol for the whole system.

This way, the inclusion of new technologies, which may appear in the future to offer new or better features, prevents the bulk of the smart-cities management system from being affected in the case of using them. In such cases, the procedure would be based, as a general rule, on creating a new module incorporating the $\mathrm{MW}$, as a driver. This module shall include the programming associated to the conversion of information formats and communication protocols. In turn, it shall allow the definition of every configuration parameter that such technology requires.

Henceforth, the inclusion of an MW with such characteristics is necessary in the system, as it means a reduction in risk of some factors that affect the conflict of decision making regarding the technology to be used in a city. In particular, the communication protocol directly affects important factors such as sustainability, adaptability and system maintenance over time.

Support to the hosting and processing of information is performed in a CC environment; therefore, such environments support this and subsequent levels. The application of CC technology, particularly to smart city management, provides the deployed system with different benefits. To begin, the initial investment gets reduced due to the fact that there is no need to acquire hardware and software to deploy the system. The flexibility is the other main advantage provided by $\mathrm{CC}$ because the service is capable of adapting itself to the demand and always providing the necessary resources. Thanks to this, the size of the city is downplayed.

Using a practical case taken from this work as an example, the MW is divided into a series of modules. Each one gets associated to a different technology. For example, to communicate through Bluetooth 4.0 (BLE - Bluetooth Low Energy), there is a module in charge of requiring the technology-specific information needed to register a sensor of this kind. For the case of BLE, this information only includes the MAC (Media Access Control) address. Once the configuration has been stored, the library Gatttool is internally used. This library makes it possible to use any BLE device through a series of simple commands. Similarly, there are specific modules to communicate through ZigBee, RFID or Wi-Fi technology, as well as through wired sensors, independently of their protocol or API. In every particular case, the specific de-encapsulation, associated to the technology, is performed. To do so, the needed information is extracted and the encapsulation to the protocol required by the higher layers in the system is performed. All these processes are performed remotely (in the CC environment), which allows us to take advantage of the benefits each technology offers regarding consumption, range or duration, without affecting the gathering of data by the higher levels of the architecture.

Thus, it is necessary to include a MW with such features in the system, as it implies the resolution of the protocol incompatibility conflict, which directly affects the stability of the entire system. However, despite using these techniques, additional conflicts still remain. 


\section{Multi-Agent System Structure}

The MAS, which is based on VO, gets deployed in the third level of the proposed architecture and constitutes the kernel of the system, where most of the efforts have been directed. It is critical to ensure this level supports tools and methodologies to provide the system with openness, reorganization and autoadaptation capabilities to certain kinds of changes in the environment. These features are highly important within MAS, which are placed in open systems such as a smart city due to their inherent characteristics (dynamism and heterogeneity). This approximation, with a MAS semi-open society, was selected as the central technology for this work as it adapts itself perfectly to the evolutionary model of a smart city.

The proposed structure is capable of solving conflicts when adding agents to a VO. Consequently, it is not only restricted to the case study in this chapter and may be used for different functionalities. However, we will focus on conflict resolution when the system needs to add a new technology.

Figure 2 shows a schema of the internal architecture of VO-based MAS, which allows the system to solve the problem of choosing the most suitable technology. These technologies are represented as external agents with the intention of entering the society to play a particular role within it. With that in mind, the presence of two or more possible technologies is said to produce a conflict between them, which needs to be solved. So, the first step consists of describing the term conflict within this work. A conflict arises when the agent society contains a set of tasks that are associated to a specific role within the MAS; the task must be satisfied by an external entity, but there are different external entities with the capability to do so. The first step is to find the External Candidate Entities (ECE) representing the

Figure 2. Proposed schema for the MAS, focused on the need for conflict resolution when choosing a sensor associated to a specific technology

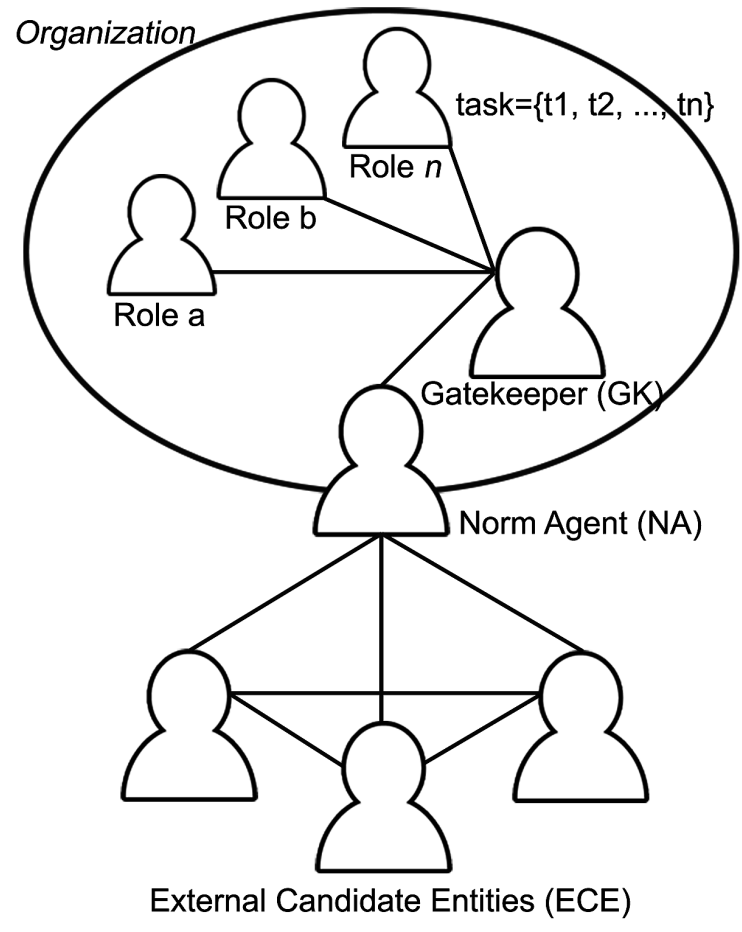


different existing options in the environment (different technologies to use), each one perfectly valid to satisfy the system's need (society) when interacting with the environment. At the beginning of the existence of a conflict, every possible solution is initially considered to be compatible with the global system. However, it is necessary to determine which ECE is the most suitable to perform the required tasks. This ECE will be integrated in the existing MAS, solving the conflict and acquiring a role within the society during a limited time needed to perform the task.

However, previously an agreement between these candidate entities must first be reached. To do so, the first step is to find out which external entities are compatible with the society. In other words, which ones comply with the norms of the society they want to enter, and in so doing will not constitute a risk in the stabilization of the MAS as a whole. The first agent they communicate with is the one with the role of Norm Agent (NA), which determines whether or not an ECE complies with the norms defined in the organization. Norms represent the obligations, constraints and qualities each agent in such an organization must somehow comply with. Each norm has an associated weight to be accumulated in a parameter to measure the incompatibility of the entity in the organization and, which should not exceed a maximum defined in the organization. For example, it guarantees that the gathered information can be communicated within the system, and does so in a stable manner, without disrupting the organization. If an ECE does not comply with the requirements, it ceases to be a candidate and will no longer take part in the negotiation process that occurs in subsequent steps.

The entities that are taken into consideration to participate in the system are considered reliable or safe, and must accept a series of factors which they must comply with to some degree. In other words, they need to be functional as well as reliable. The agent playing the Gatekeeper (GK) role is in charge of determining those factors. It does so by considering the needs of the system at any time. This agent formalizes any needs of the organization, breaking them down into a set of parameters with an associated value. This list of parameters forms the basis of negotiations the candidate entities will have to develop. The winning ECE will acquire access to the society. The value assigned to each parameter, Degree of Need $(D f N)$, is accompanied by a second value to define the priority of the parameter in question. The priority has a range of three possible nominal values depending on its grade: high (3), medium (2) and low (1).

The $D f N$ could be a positive or a negative concept depending on whether it increases or decreases. In other words, it depends on whether the fact of exceeding the value of the $D f N$ means the parameter is complying with the need or even improving the performance (an increasing parameter). On the other hand, if the $D f N$ improves when the value decreases, it is considered a negative concept. For instance, according to the example of the sensors in the city, an increasing parameter would be the sensor range because a higher range means a benefit. On the other hand, a decreasing parameter could be the consumption because the lower the consumption, the higher the autonomy or the lower the associated economic expense. These factors, which are required by the society, are communicated through the GK to different ECEs complying with the norms required by the NA. Once the requirements are received, it starts the negotiation process among the ECEs, where each one indicates to the others, in the form of an argument, the in the degree to which they provide the required factors.

A simplified way in which the entities argue the reasons why they should be chosen as part of the system is shown in Figure 3. It is based on the three following rules: 
Figure 3. State diagram to reflect the way of analyzing the rules on which the arguments of the ECEs are based

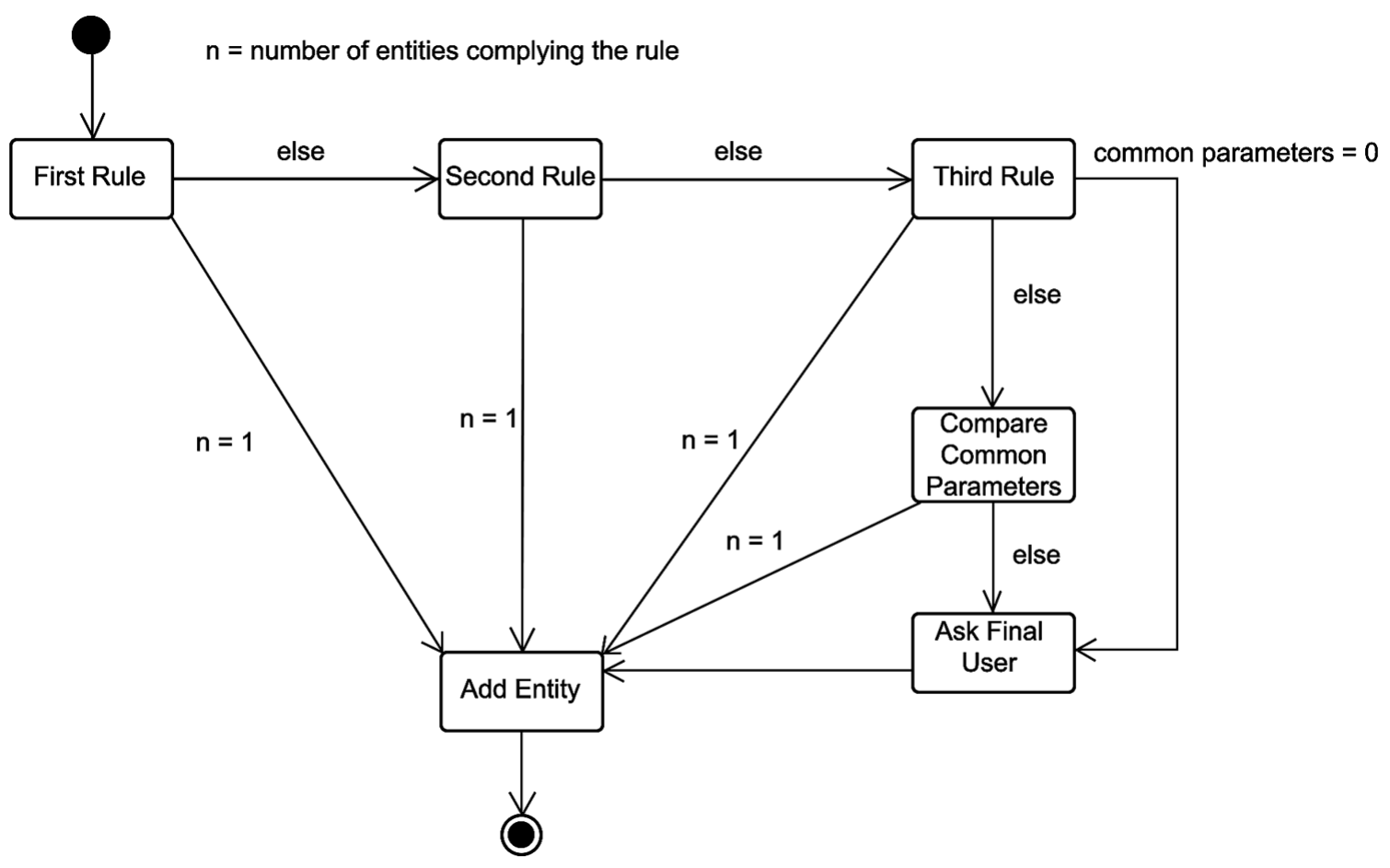

1. First Rule: Determines whether all the parameters $(R=\{1 . . n\})$ proposed by an ECE $\left(p_{i}\right)$ satisfy, in a positive way, or at least match all the parameters required by the Organization $\left(r_{i}\right)$. If only one candidate entity fulfills the rule, it will enter the system. When two or more ECEs fulfill the rule, these entities will evaluate the following rule. Finally, when no entity satisfies the first rule, every candidate entity moves on to evaluate the second rule.

$\forall i \in R / p_{i}-r_{i} \geq 0, R=\{1 . . n\}$

2. Second Rule: Calculates the degree to which the parameters $(R=\{1 . . n\})$ proposed by each ECE $\left(p_{i}\right)$ solve the requirements of the system $\left(r_{i}\right)$. If the greatest value obtained is associated to a single entity, this entity will become part of the system. But, if two or more entities share the obtained maximum value, they move on to evaluate the following rule.

$\sum_{i=1}^{n}\left(p_{i}-r_{i}\right)$ 
3. Third Rule: The concept of the priority of each parameter $\left(y_{i}\right)$ is considered when evaluating this rule. Following the logic of the previous rule, the entity with the maximum value will enter the system.

$$
\sum_{i=1}^{n}\left(\left(p_{i}-r_{i}\right) \cdot y_{i}\right)
$$

If as a result of the last rule, there appears to be an entity plurality, there are two possible solutions. The first solution compares the values of the not required by the system but common to those entities parameters, arguing the reason that it should be the chosen entity. When it is not possible to solve the conflict, the system autonomously asks the final user to select a decision among those the system has already pre-selected.

\section{Services}

The services offered by the architecture are based on offering multiple possibilities to the end user. These possibilities range from monitoring the state of the whole system, where the state of each element participating in the system can be observed, to individually or collectively controlling such elements.

This type of control can be performed by defining a series of guidelines for the system to autonomously be able to replicate them when in similar situation. These guidelines to follow may be, for example, based on data mining or any other Artificial Intelligence (IA) technology to maximize the energetic efficiency of public lighting in the city.

Entrance services are also provided for the elements to be registered and for the existing possibilities to be presented. The conflict resolution is described and the most efficient solution for the system is provided to the end user. It is precisely at this moment that the city begins to achieve efficiency.

\section{Study Case and Preliminary Evaluation}

One example of applying this approach to solving conflicts in the proposed case study is traffic management. Sensors with different functionalities participate during the performance of this task. For example, an organization with this purpose could be called "Traffic Organization". In this organization, there are different types of roles apart from the GK role and the NA role. In this case, there are agents to obtain information (vehicle-counting role, pedestrian flow detection role, etc.), decision-making roles for the criteria related to traffic (size of roads, timetables, information gathered by the sensors, etc.) and actuators (role in charge of modifying the state of the traffic lights).

The feature of information acquisition and actuation is directly related to that of technology, for which this work attempts to offer a solution (we are not taking into consideration the AI issues related to decision making).

As an example, when adopting a technology as a solution to the vehicle-counting role, a case is presented with the following possibilities (real alternatives are simplified to facilitate the comprehension of the example): 
- Sensor on the Road: Pressure sensors are deployed along the roads (underneath the asphalt or with bands adhered to it). The following technologies are available for this kind of sensor:

- Bluetooth.

$\circ \quad$ RFID.

- ZigBee.

- 6lowpan.

- Optical IP Camera: It may be placed over a traffic light or on the side of the road. It allows the following technologies

$\circ$ Wi-Fi.

- Ethernet (with Power over Ethernet).

For this particular example, the system will only require three parameters $(D f N)$. The parameters chosen for our consideration are:

- Range: A minimum range of 10 meters is required so that it can be assigned the value 10. High priority, as it is mandatory to guarantee the information can arrive from one side of the road to the other, in case an intermediate node fails. It is an increasing parameter.

- Consumption: The sensors should not drain more than $200 \mathrm{~mA}$ as an average when transmitting. Medium priority, as a lower priority than the range priority is assigned. It is a decreasing parameter so its value is negative.

- Price: The price of the sensors for smart cities is a relevant factor to be taken into consideration because of the large number of sensors to be used in a smart city. For our case, a maximum price of $40 €$ per unit is established, taking into account the criteria based on the city budget. If the main idea is to save money, we should not discount the installation costs. Medium priority. It is a decreasing parameter (negative values).

Clearly there are more influencing parameters, such as the deployment cost and maintenance cost, but again, a reduced view is provided for a better understanding. Table 1 shows the values for each of the candidate entities vying to become part of the system.

Table 1. Candidate technologies and values provided for the system degree of need

\begin{tabular}{|l|c|c|c|}
\hline \multicolumn{1}{|c|}{ Technology } & Range & Consumption & Price \\
\hline Bluetooth 4.0 & $10 \mathrm{~m}$ & $25 \mathrm{~mA}$ & $30 €$ \\
\hline RFID & $30 \mathrm{~m}$ & $24 \mathrm{~mA}$ & $40 €$ \\
\hline ZigBee & $15 \mathrm{~m}$ & $30 \mathrm{~mA}$ & $30 €$ \\
\hline 6lowpan & $60 \mathrm{~m}$ & $24 \mathrm{~mA}$ & $20 €$ \\
\hline Wi-Fi & $50 \mathrm{~m}$ & $1000 \mathrm{~mA}$ & $80 €$ \\
\hline Ethernet & $100 \mathrm{~m}$ & $600 \mathrm{~mA}$ & $70 €$ \\
\hline
\end{tabular}


Table 2. Arguing table for each candidate technology

\begin{tabular}{|l|c|c|c|}
\hline \multicolumn{1}{|c|}{ Technology } & First Rule & Second Rule & Third Rule \\
\hline Bluetooth 4.0 & $\mathbf{2 0} ; \geq \mathbf{0} ; \geq \mathbf{0}$ & 185 & 370 \\
\hline RFID & $\mathbf{2 0} ; \geq \mathbf{0} ; \geq \mathbf{0}$ & 196 & 412 \\
\hline ZigBee & $\mathbf{0} ; \geq \mathbf{0} ; \geq \mathbf{0}$ & 185 & 375 \\
\hline 6lowpan & $\geq \mathbf{0} ; \geq \mathbf{0} ; \geq \mathbf{0}$ & $\mathbf{2 4 6}$ & $\mathbf{5 4 2}$ \\
\hline Wi-Fi & $\mathbf{2 0} ;<0 ;<0$ & -800 & -1600 \\
\hline Ethernet & $\mathbf{2 0} ;<0 ;<0$ & -340 & -590 \\
\hline
\end{tabular}

Assuming that every entity complies with the access norms to the system, each one will use the values described in Table 2 when arguing its case for accessing the system.

Based on the arguing criteria, the entity that will become part of the system following the vehiclecounting role from the "Traffic Organization" is, in this case, the entity associated to the 6lowpan technology. This is because it has the highest value in the application of the second rule.

One of the main disadvantages of the 6lowpan technology is that it is not very widespread among users, which means the selection of the parameters to be considered is very important. For instance, had we only used an extra parameter, in addition to the other three required parameters, to refer to support, it is possible that the 6lowpan would not have been the chosen technology. In order to guarantee the technological decisions are adequate, we suggest analyzing, apart from those already used, at least the following parameters: maintenance cost, deployment cost, upgradability and maximum size of the network.

To test the proper performance of the architecture, the system was applied to the simulation environment of a smart city, as a preliminary evaluation. This environment recreates the main characteristic elements of this kind of city in a model of a city. The model was almost entirely 3D printed, having an area of $2 \times 2$ meters. This model can be seen in Figure 4. By using this architecture as the base, it is possible to manage every element on the model: luminaries, solar panels, traffic lights, containers,

Figure 4. Simulation environment used to develop the architecture testing (left), along with the management software and monitoring of the smart city (right)

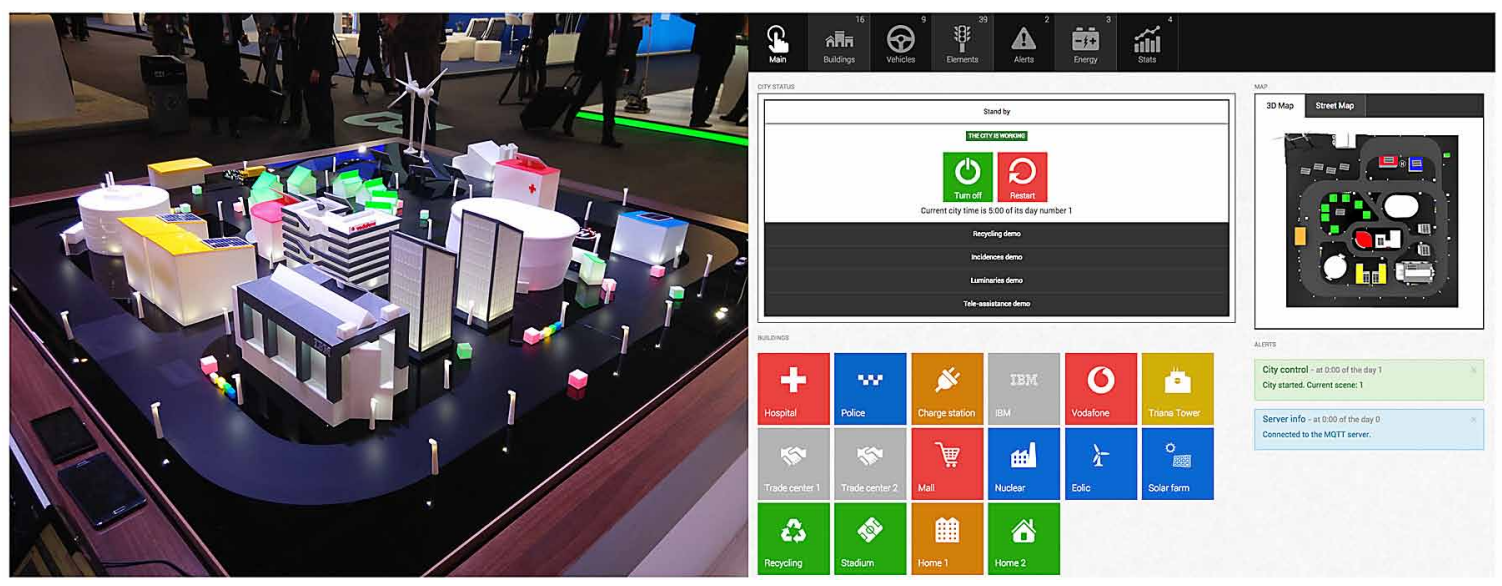


aero-generators and even vehicles. This way, although the number of sensors is lower than in a real smart city, some testing on the offered services, such as the decision making when choosing the sensor to be used, was developed

\section{FUTURE RESEARCH DIRECTIONS AND CONCLUSION}

This work has created a platform capable of solving conflicts, with a special focus on smart cities management. The problems in this study have focused on technology used as a base to gather and merge the information of everything happening on it, as it is the first conflict posed when designing a platform with such features.

Nevertheless, the schema can be extrapolated to superior levels in the architecture where decisions are taken to a higher level. For instance, for reaching an agreement and achieving conflict resolution, it is possible to apply the proposed schema to the distribution of street lighting between the streets in a city, taking multiple factors into account (pedestrian flow, traffic, location, businesses, etc.). The efforts made have not quite finished and more efforts will be made in order to apply the system to new case studies within the smart city environment.

In the long term, the results of the architecture can be measured by quantifying the savings produced in a city using this system, as opposed to other cities with similar characteristics (size, number of citizens, institutions and number of sensors) but a different management system.

Moreover, this system is not just limited to smart cities. For example, it could be extrapolated as a system to manage data from a WSN in a residential home, in addition to many other fields of application. Therefore, it is possible to continue applying the architecture to different environments, which would allow its functionality to increase.

This work has presented a platform capable of solving conflicts in the management of smart cities, which, and has focused on the technology to be used as a base to gather and merge information regarding all events occurring on it. This feature is practically vital when centralizing all activities taking place in a city into a stable system within a project with continuity. While a standardized and common sensor protocol is not presented, the offered solution remains an alternative capable of isolating the part of communication with the sensor from the result of the global system. The platform can also adapt itself to changes in technology when the existing system becomes more inefficient, which permits making the most of the advantages coming from the currently offered technologic diversity and the benefit of gathering the information in a unique manner.

\section{ACKNOWLEDGMENT}

This work has been supported by the European Commission H2020 MSCA-RISE-2014: Marie SkłodowskaCurie project DREAM-GO Enabling Demand Response for short and real-time Efficient And Market Based Smart Grid Operation - An intelligent and real-time simulation approach ref 641794 


\section{REFERENCES}

Akyildiz, I. F., \& Vuran, M. C. (2010). Wireless sensor networks (Vol. 4). John Wiley \& Sons. doi:10.1002/9780470515181

Annunziato, M., \& Pierucci, P. (2003). The emergence of social learning in artificial societies. In Applications of evolutionary computing (pp. 467-478). Springer Berlin Heidelberg. doi:10.1007/3-54036605-9_43

Armbrust, M., Fox, A., Griffith, R., Joseph, A. D., Katz, R., Konwinski, A., \& Zaharia, M. et al. (2010). A view of cloud computing. Communications of the ACM, 53(4), 50-58. doi:10.1145/1721654.1721672

Buratti, C., Conti, A., Dardari, D., \& Verdone, R. (2009). An overview on wireless sensor networks technology and evolution. Sensors (Basel, Switzerland), 9(9), 6869-6896. doi:10.3390/s90906869 PMID:22423202

Caragliu, A., Del Bo, C., \& Nijkamp, P. (2011). Smart cities in Europe. Journal of Urban Technology, 18(2), 65-82. doi:10.1080/10630732.2011.601117

Chopra, A. K., \& Singh, M. P. (2013). Multiagent Systems: A Modern Approach to Distributed Artificial Intelligence. In Agent Communication (pp. 101-141). The MIT Press.

Daft, R. (2012). Organization theory and design. Cengage Learning.

Davidsson, P., \& Johansson, S. (2006). On the potential of norm-governed behavior in different categories of artificial societies. Computational \& Mathematical Organization Theory, 12(2-3), 169-180. doi:10.1007/s10588-006-9542-x

De la Prieta, F., Rodríguez, S., Bajo, J., \& Corchado, J. M. (2013). A multiagent system for resource distribution into a Cloud Computing environment. In Advances on Practical Applications of Agents and Multi-Agent Systems (pp. 37-48). Springer Berlin Heidelberg. doi:10.1007/978-3-642-38073-0_4

Ferber, J. (1999). Multi-agent systems: an introduction to distributed artificial intelligence (Vol. 1). Reading: Addison-Wesley.

Ferber, J., Gutknecht, O., \& Michel, F. (2004). From agents to organizations: an organizational view of multi-agent systems. In Agent-Oriented Software Engineering IV (pp. 214-230). Springer Berlin Heidelberg. doi:10.1007/978-3-540-24620-6_15

Hafeez, A., Kandil, N. H., Al-Omar, B., Landolsi, T., \& Al-Ali, A. R. (2014). Smart Home Area Networks Protocols within the Smart Grid Context. Journal of Communication, 9(9).

Hollands, R. G. (2008). Will the real smart city please stand up? Intelligent, progressive or entrepreneurial? City, 12(3), 303-320. doi:10.1080/13604810802479126

Lian, K. Y., Hsiao, S. J., \& Sung, W. T. (2013). Intelligent multi-sensor control system based on innovative technology integration via ZigBee and Wi-Fi networks. Journal of Network and Computer Applications, 36(2), 756-767. doi:10.1016/j.jnca.2012.12.012 
Liu, Y., Seet, B. C., \& Al-Anbuky, A. (2013). An ontology-based context model for Wireless Sensor Network (WSN) management in the internet of things. Journal of Sensor and Actuator Networks, 2(4), 653-674. doi:10.3390/jsan2040653

Mitton, N., Papavassiliou, S., Puliafito, A., \& Trivedi, K. S. (2012). Combining Cloud and sensors in a smart city environment. EURASIP Journal on Wireless Communications and Networking, (1): 1-10.

Navarro, M., Bhatnagar, D., \& Liang, Y. (2011, October). An integrated network and data management system for heterogeneous WSNs. In Mobile Adhoc and Sensor Systems (MASS), 2011 IEEE 8th International Conference on (pp. 819-824). IEEE. doi:10.1109/MASS.2011.94

Roscia, M., Longo, M., \& Lazaroiu, G. C. (2013, October). Smart city by multi-agent systems. In Renewable Energy Research and Applications (ICRERA), 2013 International Conference on (pp. 371-376). IEEE. doi:10.1109/ICRERA.2013.6749783

Schaffers, H., Komninos, N., Pallot, M., Trousse, B., Nilsson, M., \& Oliveira, A. (2011). Smart Cities and the Future Internet: Towards Cooperation Frameworks for Open Innovation. Future Internet Assembly, 6656, 431-446. doi:10.1007/978-3-642-20898-0_31

Talia, D. (2012). Clouds meet agents: Toward intelligent cloud services. IEEE Internet Computing, 16(2), 78-81. doi:10.1109/MIC.2012.28

Vasconcelos, W. W., Kollingbaum, M. J., \& Norman, T. J. (2009). Normative conflict resolution in multi-agent systems. Autonomous Agents and Multi-Agent Systems, 19(2), 124-152. doi:10.1007/ s10458-008-9070-9

Weber, R. H. (2010). Internet of Things-New security and privacy challenges. Computer Law \& Security Report, 26(1), 23-30. doi:10.1016/j.clsr.2009.11.008

Zambonelli, F., Jennings, N. R., \& Wooldridge, M. (2001). Organisational rules as an abstraction for the analysis and design of multi-agent systems. International Journal of Software Engineering and Knowledge Engineering, 11(03), 303-328. doi:10.1142/S0218194001000505

Zato, C., De Paz, J. F., de Luis, A., Bajo, J., \& Corchado, J. M. (2012). Model for assigning roles automatically in egovernment virtual organizations. Expert Systems with Applications, 39(12), 10389-10401. doi:10.1016/j.eswa.2012.01.185

\section{KEY TERMS AND DEFINITIONS}

Agent: In a global and reduced way, as there are loads of versions to define the term as well as possible classifications characteristics to comply with, it is a physical or virtual entity that acts by responding to collected stimuli from its environment. This response needs to be rational, tending to maximize the desired result.

Cloud Computing: It is a kind of computation where all the resources from a computational system are offered as a service through the Internet. This way, the demander gets unlinked from the way those resources are managed, focusing only in its use. So the user does not need any knowledge on the way those resources need to be managed, saving time, and in many occasions money. 
Gatekeeper: It defines an access point to organizations in semi-open type of MAS trough which all the external entities have to contact in order to enter the society. Its purpose is to guarantee every entity in the system complies with a degree of security. This is what guarantees it is a reliable and stable system even when having some degree of openness.

Multi-Agent System: It is composed of agents and their environment. This kind of systems is usually used to solve problems that are difficult or even impossible for single agents to solve.

Role: It defines the position and the set of responsibilities an agent within the global system has. An agent is capable of adopting different roles depending on the context.

Smart City: It is an emerging term during the last decade. It represents the concept of efficient and sustainable city, capable of responding in the most adequate way to the basic needs of the city. This response to the needs is mainly based on the economic, environmental, social and operative fields. To do so, an efficient and lasting infrastructure needed to manage water, energy, tele-communications, transportation, emergency services, etc.

Virtual Organization: It could be seen as a set of roles with a certain relationship among them, with the capability of interacting with other roles trough certain patterns. They are a way of interpreting the MAS from a sociologic point of view.

Wireless Sensor Network: It is a spatially distributed network of autonomous sensors used to monitor physical or environmental conditions, for example, in a smart city. In general, they are composed of a series of nodes connected together in a way that the network allows knowing the information each one of those nodes gathers. 